\title{
Are protozoan metacaspases potential parasite killers?
}

\author{
Benoît Meslin ${ }^{1}$, Habib Zalila², Nicolas Fasel ${ }^{2}$, Stephane Picot ${ }^{1}$, Anne-Lise Bienvenu ${ }^{1 *}$
}

\begin{abstract}
Mechanisms concerning life or death decisions in protozoan parasites are still imperfectly understood. Comparison with higher eukaryotes has led to the hypothesis that caspase-like enzymes could be involved in death pathways. This hypothesis was reinforced by the description of caspase-related sequences in the genome of several parasites, including Plasmodium, Trypanosoma and Leishmania. Although several teams are working to decipher the exact role of metacaspases in protozoan parasites, partial, conflicting or negative results have been obtained with respect to the relationship between protozoan metacaspases and cell death. The aim of this paper is to review current knowledge of protozoan parasite metacaspases within a drug targeting perspective.
\end{abstract}

\section{Metacaspases: a twenty-first century history}

In the late nineties, Aravind from Bethesda was the first to describe orthologues of caspases [1]. This paved the way for Uren and colleagues to describe paracaspases from animals and slime mould, and metacaspases from plants, fungi and protozoa, in the beginning of $21^{\text {st }}$ century [2]. Caspases are limited to metazoans, while metacaspases are missing from them: leading to the hypothesis that metacaspases resemble ancestral proteases and that caspases have diverged through evolution under environmental stresses. Approximately a hundred papers were published during the first decade of the new century: mostly studying the role of metacaspase in apoptosis in the budding yeast, Saccharomyces cerevisiae, for example $[3,4]$. While the involvement of yeast metacaspase in cell death is well documented [5,6], a non-death role for the yeast metacaspase Yca1p has also been described [7]. More recently, authors have strongly expressed their disagreement with the classification of metacaspases as part of the caspases family [8]. It was argued that the specificity of these enzymes should determine their classification, rather than any similarities. Other papers with definitive titles ("Are metacaspases caspases?" [9] and "Metacaspases are caspases. Doubt no more" [10]) were published, demonstrating the vitality of the debate. Whether this controversy will address issues of medical importance is debatable.

\footnotetext{
* Correspondence: anne-lise.bienvenu@recherche.univ-lyon1.fr

'Malaria Research Unit, University Lyon 1, 8 Avenue Rockefeller, 69373 Lyon cedex 08, France

Full list of author information is available at the end of the article
}

However, it demonstrated the requirement to explore the role of metacaspases, as an aid to determining whether renaming these enzymes in agreement with their definitive specificity is needed.

Although only recently described and their function poorly explored, metacaspases could be considered as potential targets for new specific treatments against the main protozoan parasites affecting humans. Elucidating their role in physiology is certainly a requirement, but it is clear that addressing this issue could provide new insights into the fight against tropical diseases.

\section{Structure/Activity of metacaspases}

Peptidases are classified in the MEROPS database http:// merops.sanger.ac.uk using "clans" and "families" features. All the sequences in the same clan are evolutionarily-related, determined by similarities in protein tertiary structures and share a similar protein fold [11]. A peptidase clan consists of two letters, the first designating the catalytic type, for example A for aspartic acid, C for cysteine, $\mathrm{S}$ for serine. Because some cysteine, serine and threonine peptidases have similar folds, the letter " $\mathrm{P}$ " is used for clans of mixed catalytic type. Among the peptidases forming a transient covalent bond between the substrate and the enzyme, serine, threonine and cysteine peptidases are well described [12].

Metacaspases (MCA) are cysteine proteases with structural similarity to caspases and a catalytic cysteinehistidine dyad. Caspases and metacaspases are members of the C14 family, clan CD, with probable differences in 
substrate specificity [13]. Surprisingly, it was recently demonstrated that two different type 1 metacaspases from a plant (Arabidopsis thaliana) differentially regulate superoxide-dependant cell death: AtMC1 is a prodeath caspase-like protein while AtMC2 antagonizes this function [14]. Metacaspases have a highly acidic S1 pocket leading to a basic specificity for arginine and lysine at the P1 position, rather than aspartic acid specificity as seen for caspases [12,15].

Differences in substrate specificity between metacaspases and caspases lead to uncertainty concerning the potential role of protozoan metacaspases in apoptosislike cell death, and it has been proposed that metacaspases and paracaspases should be separated in a specific family in clan CD [9]. Nevertheless, it was recently demonstrated that caspases and metacaspases can both target the Tudor Staphylococcal Nuclease (TSN) [16]. TSN is an evolutionary conserved protein, composed of a single Tudor domain and five staphylococcal nucleaselike domains, and is involved in a number of transcriptional and post-transcriptional pathways. Homo sapiens TSN sequence shows a consensus cleavage site for caspase-3, and proteolysis of TSN is blocked after treatment with the pan-caspase inhibitor zVAD-fmk. Although the DAVD caspase- 3 cleavage motif is absent from protozoa, fungi and plants, TSN proteins from the plant Picea abies were found to contain several metacaspase cleavage sites [16]. The ability of caspase from vertebrate and type 2 metacaspase from plant to cleave the same substrate with a central role in the cell degradome provides new evidence that metacaspase could be involved in apoptosis control [16].

\section{Plasmodium Metacaspases}

While insecticide-treated bednets, Artemisinin-based combined therapy (ACTs) and climatic changes have transformed the global impact of malaria in endemic areas, this parasitic disease remains a major life-threatening challenge for millions of people. ACTs are the drugs of choice for non-severe malaria, but parasite resistance is speculated to appear in the near future [17]. Thus, it is of utmost importance to decipher the mechanisms of parasite growth control, in order to detect new drug targets; potentially not subjected to resistance. Apoptosis-like DNA fragmentation of Plasmodium falciparum parasite in response to chloroquine treatment was described more than a decade ago [18]. It was speculated that resistance to chloroquine was related to inhibition of apoptosis. Plasmodium berghei features of apoptosis including condensation of chromatin, DNA fragmentation and externalisation of phosphatidylserine were demonstrated a few years later [19]. Proteins with caspase-like activity were identified in the cytoplasm of the ookinete, and more than $50 \%$ of the mosquito midgut stages of the parasite die naturally by apoptosis before gut invasion. This phenomenon was prevented by a caspase inhibitor [19].

Metacaspase, as well as clans CA, CD and CE proteases have been described in Plasmodium [20]. Clan CA proteases are the best-characterised cysteine proteases of Plasmodium (Table 1). The major functions of clan CA proteases, including falcipains, concerned with hemoglobin hydrolysis and erythrocyte rupture or invasion was extensively reviewed a few years ago [21]. However, few studies have been conducted to decipher the role of Plasmodium metacaspases [22,23].

Three MCAs were described in the genome of the two major human malaria parasites, P. falciparum (PfMCA13 ) and P. vivax (PvMCA1-3), and in the murine parasite P. berghei (PbMCA1-3). PxMCA1 is the only enzyme that presents the well-characterized histidine and cysteine catalytic dyad. Patterns of expression seem to differ between PfMCA1 and PbMCA1. PfMCA1 gene expression was described in the erythrocytic stage [Meslin et al, unpublished observations], PbMCA1 expression was detected in female gametocytes, in oocysts and in sporozoites, but authors [23] failed to demonstrate a significant level of ookinetes apoptosis compared to other studies [19,24], and did not observe differences between wild-type (WT) and PbMCA1-KO ookinetes, leading to the conclusion that functional redundancy may exist, probably through $\mathrm{PbMCA} 2 / 3$ activity. It should be kept in mind that apoptosis of normal parasites may occur in specific conditions that lead to a message of death. A more comprehensive study of the role of metacaspase in Plasmodium biology should be conducted in the presence of one such message. In a recent study, Plasmodium falciparum metacaspase expression was measured during parasite culture in vitro, showing a relationship with parasitemia levels and suggesting a role in growth regulation [25]. These preliminary results provide some more evidence for a role of metacaspase in Plasmodium fitness.

The expression of PfMCA1 C14 peptidase domain in $y c a 1$ deficient $S$. cerevisiae led to growth retardation and a drastic yeast cell death [Meslin et al, unpublished observations]. Interestingly, this phenotype could be blocked by the addition of the pan caspase inhibitor z-VAD-fmk while PfMCA1 did not exhibit a caspase-like, but an arginine protease activity, as reported for other protozoan MCAs [Meslin et al, unpublished observations]. PfMCA1 could play an initiator role leading to the activation of an aspartate protease effector. This hypothesis is in agreement with the description of PfMCA1 autoprocessing leading to prodomain removal as typical of initiator caspases [22]. PfMCA1 function could to be regulated by the two putative binding domains described: a $\mathrm{C} 2$ calciumdependant membrane targeting domain and a CARD 


\begin{tabular}{|c|c|c|c|}
\hline & Structure, processing and enzymatic activity & Expression, localisation and function & References \\
\hline PbMCA1 & $\begin{array}{l}\text { Absence of caspase-like protease activity. } \\
\mathrm{C} 2 \text { domain in N-terminus. } \\
\mathrm{P} \text { replaces the predicted catalytic C but a C is located } \\
\text { immediately adjacent. }\end{array}$ & $\begin{array}{l}\text { Expressed only in female gametocytes and all } \\
\text { downstream mosquito stages. } \\
\text { Absence of loss-of-function in PbMCA1 deficient parasites. }\end{array}$ & [23] \\
\hline PfMCA1 & $\begin{array}{l}\text { C2 and putative CARD domains in N-terminus. } \\
\text { Autoprocessing upstream the C14 peptidase domain. } \\
\text { R-specific and calcium-dependant proteolytic activity. } \\
\text { Absence of caspase-like protease activity. }\end{array}$ & $\begin{array}{l}\text { Expressed in erythrocytic stages and mainly in the } \\
\text { schizont. } \\
\text { Induced yeast cell death and growth retardation when } \\
\text { over-expressed in } \Delta y c a 1 \text { yeasts treated with } \mathrm{H}_{2} \mathrm{O}_{2} \text {. }\end{array}$ & {$[20]$} \\
\hline $\begin{array}{l}\mathrm{PfMCA} 2 / 3 \\
\mathrm{PbMCA} 2 / 3\end{array}$ & $\begin{array}{l}\text { Absence of the } \mathrm{H} / \mathrm{C} \text { catalytic dyad but a } \mathrm{C} \text { is located one } \\
\text { residue upstream the consensus } \mathrm{C} \text { for PfMCA3. }\end{array}$ & & {$[22,23]$} \\
\hline
\end{tabular}

C2 domain: calcium-dependent membrane targeting motif. Abbreviations: BSF, bloodstream form; PCF, procyclic form; MCA, metacaspase; $C$, cysteine; $D$, aspartic acid; $\mathrm{H}$, histidine; $\mathrm{K}$, lysine; $\mathrm{N}$, asparagine; $\mathrm{P}$, proline; $\mathrm{R}$, arginine; $\mathrm{S}$, serine; $\mathrm{W}$, tryptophan; FHS, fresh human serum; MLS, mitochondrial localisation signal; CARD, caspase recruitment domain.

domain (Caspase Recruitment Domain) [22,23]. Interestingly, Plasmodium falciparum Tudor Staphylococcal Nuclease was recently described and some of its interacting proteins were detected using two-hybrid analysis, colocalization and co-immunoprecipitation [26,27]. Plasmodium Tudor SN exhibits RNA binding and nuclease activity. Whether Plasmodium Tudor SN could be a substrate for metacaspase is still unknown, but it probably has a central role in the parasite life cycle.

\section{Trypanosomatids Metacaspases}

The kinetoplastid protozoan, Trypanosoma brucei, Trypanosoma cruzi and several Leishmania species cause, respectively, sleeping sickness, Chagas disease and various forms of leishmaniasis $[28,29]$. Together, these pathogenic agents affect millions of people across the world, especially in developing countries, where they are often totally mismanaged (WHO/TDR, http://www.who. int/tdr). Metacaspases have been identified and partially described in all trypanosomatids and with the recent discovery of anti-metacaspase inhibitors, further research in this area may prove to be clinically significant.

\section{1 - Trypanosoma}

In the African trypanosome, T. brucei, five genes encoding metacaspases (TbMCA1-TbMCA5) are expressed (Table 2). At the protein level, TbMCA2 and TbMCA3 are highly similar ( $89 \%$ of sequence identity), with the difference ascribed to putative organelle targetingdomain at the $\mathrm{N}$-terminus of TbMCA3. TbMCA5 has a central domain, typical of a metacaspase, as well as an additional C-terminal extension [13]. Importantly, TbMCA1 and TbMCA4 have amino-acid substitutions in their conserved metacaspase catalytic residues (histidine to tyrosine and cysteine to serine in TbMCA1; cysteine to serine in TbMCA4) and are therefore expected to be enzymatically inactive [13].

Metacaspases have also been studied in their physiological context, TbMCA2 and TbMCA3 are reportedly expressed only in the bloodstream form of the parasite whereas TbMCA5 is detectable throughout the parasite life cycle, where they appear to play a role in the recycling of endosomes co-localising with RAB11 in recycling vesicles [30]. Further, sequential genetic deletion of TbMCA2, TbMCA3 and TbMCA5 did not lead to cell death and even triple knockout parasites were able to recover growth rates comparable to wild-type cells. On the other hand, a rapid down-regulation of their expression using RNA-interference led to a pre-cytokinesis cell-cycle blockade; perhaps because there was inefficient time for the hypothesized compensation [13]. All five metacaspases have been studied in the heterologous organism S. cerevisiae, where only TbMCA4 overexpression resulted in growth inhibition and cell death. Interestingly, it was shown to be localised in the nucleus and appeared to play a role at the mitochondrial level by inducing a loss of respiratory competence in yeast cells [31].

TbMCA2 has been further biochemically characterised; it has arginine/lysine specificity at substrate P1 position [32] similar to plant metacaspases $[9,33]$. Despite the fact that it is auto-cleaved at lysine positions, this processing does not seem to be important for its enzymatic activity. TbMCA2 also showed a strict calcium dependency that could reach $1 \mathrm{mM}$ for maximum activity; a biological concentration only encountered in acidocalcisomes. However, no experimental evidence has yet linked TbMCA2 to these compartments [32].

So far, T. brucei metacaspases (especially TbMCA2, TbMCA3 and TbMC5) appear to be essential in the bloodstream form of the parasite [30] and could have potential as clinically viable drug targets to fight sleeping sickness.

T. cruzi expresses two metacaspase proteins, one homologous to TbMCA2 (named TcMCA3) and a second homologous to TbMCA5 (named TcMCA5) [34]. The TCMCA3 gene is present in 16 copies per haploid genome and the encoded protein has been found to be 
Table 2 Key features on Trypanosoma metacaspases

\begin{tabular}{|c|c|c|c|}
\hline & Structure, processing and enzymatic activity & Expression, localisation and function & References \\
\hline TbMCA1/4 & $\begin{array}{l}\text { S replaces the predicted catalytic } C \text { but a } C \text { is } \\
\text { located immediately adjacent. } \\
\text { Absence of peptidase activity after R/D/N/K when } \\
\text { TbMCA4 was expressed in S. cerevisiae or in E. coli. }\end{array}$ & $\begin{array}{l}\text { TbMCA4 induced mitochondrial dysfunction, clonal death and } \\
\text { growth inhibition when expressed in S. cerevisiae. } \\
\text { Nuclear localisation for TbMCA4. }\end{array}$ & {$[13,31]$} \\
\hline TbMCA2/3/5 & $\begin{array}{l}\text { No processing in vivo but autoprocessing for } \\
\text { TbMCA2 after K55 and K268 in E. coli with calcium. } \\
\text { Class III WW domain in N-terminus of TbMCA2/3 } \\
\text { and in C-terminus of TbMCA5. } \\
\text { TbMCA2 proteolytic activity: R/K-specific, Ca }{ }^{2+} \text { dose- } \\
\text { dependent and processing not required. } \\
\text { Sensitive to leupeptin, antipain, TLCK and } \\
\text { component with R in P1 position but insensitive to } \\
\text { E64 inhibitor. }\end{array}$ & $\begin{array}{l}\text { Expression in the BSF but not in the PCF for TbMCA2/3 and in } \\
\text { both forms for TbMCA5. } \\
\text { No loss-of-function phenotype detected for the triple null } \\
\text { mutants ( } \triangle \text { mca2/3 } \triangle \text { mca5). } \\
\text { Triple RNAi induced a rapid growth arrest and a dysregulation } \\
\text { of cytokinesis. } \\
\text { Colocalisation of TbMCA2/3/5 with RAB11-positive endosome } \\
\text { but no further data support involvement of TbMCAs in cell } \\
\text { trafficking. }\end{array}$ & {$[30,44]$} \\
\hline ТсMCA3/5 & $\begin{array}{l}\text { TCMCA3 gene is present in } 16 \text { copies and TCMCA5 in } \\
\text { a single copy in the T. cruzi genome. } \\
\text { Q/P/Y rich region in the C-terminus of TCMCA5. } \\
\text { Caspase-like activity concomitant with TCMCA3 } \\
\text { nuclar relocalisation upon (FHS)-induced cell death. }\end{array}$ & $\begin{array}{l}\text { Expression of TCMCA5 in epimastigote form. } \\
\text { Expression of TCMCA3 in the four major stages of development. } \\
\text { Cytoplasmic localisation and migration into the nucleus upon } \\
\text { FHS treatment. } \\
\text { Epimastogotes over-expressing TCMCA5 are more sensitive to } \\
\text { (FHS)-induced cell death. }\end{array}$ & [34] \\
\hline
\end{tabular}

expressed during the entire parasitic life cycle, whereas TCMCA5 is present as a single copy and the expressed protein is only found in the epimastigote form of the parasite. Attempts to biochemically characterize these MCAs have been unsuccessful, however Kosec et al. were able to show that TcMCAs could be involved in the apoptosis of the parasite as they found them to relocalise from the cytoplasm to the nucleus during apoptosis induced by fresh human serum [34]. A further caspase-like activity was reported and is most likely due to a downstream protease.

Five studies have been conducted during the last decade to explore the presence and the role of metacaspases in Trypanosoma brucei and T. cruzi parasites in vitro. While interesting information was obtained on similarities between Trypanosoma metacaspases and caspase families, proof that metacaspase activity could be similar to caspase and involved in apoptosis-like cell death is still lacking.

\section{2 - Leishmania}

All Leishmania species express one single metacaspase gene except in infantum and donovani subtypes where two metacaspases have been found encoded as GeneDB http://www.genedb.org. Leishmania metacaspases have a high sequence homology (77.4 to 99.8\%). Structurally, they share an $\mathrm{N}$-terminal domain containing a putative mitochondrial-localisation signal, a central metacaspase domain (containing the conserved catalytic dyad histidine and cysteine) and a less conserved proline-rich C-terminal domain (61.4 to $100 \%$ homology), which probably plays a role in protein-protein interactions [35] (Table 3).

In L. donovani, LdMC1 and LdMC2 were reportedly expressed in both promastigote and axenic amastigote forms of the parasite. They exhibit an arginine/lysine- specific activity without any requirement for proteolytic activation, neither in normal conditions nor upon oxidative stress-induced PCD. LdMCs have been found to be localised in the acidocalcisomes where they have been purportedly sequestered as inactive enzymes which are released upon apoptosis induction using oxygen peroxide $\left(\mathrm{H}_{2} \mathrm{O}_{2}\right)$, as measured by the increase in metacaspase activity and TUNEL positive cells [36]. In L. major, the single metacaspase (LmjMCA) was found to be able to replace yeast metacaspase (YCA1) in the $\mathrm{H}_{2} \mathrm{O}_{2}$-induced death phenotype of S. cerevisiae [35]. Although it has similar activities towards arginine and lysine, in contrast to LdMCs LmjMCA was reported to be activated by autoprocessing and the purified putative catalytic domain was 300 times more active than the nonpurified full-length LmjMCA [35].

The $\mathrm{H}_{2} \mathrm{O}_{2}$-induced death role of LmjMCA has been further characterized within the parasite. LmjMCA overexpression was found to enhance $L$. major sensitivity to oxidative stress as measured by the increase of phosphatidylserine exposure at the parasites surface and the rapid loss of mitochondrial membrane potential as compared to wild-type parasites expressing the endogenous metacaspase at physiological levels. LmjMCA was found to be extensively processed and the catalytic domain and accumulated in the cytosolic subcellular fraction when apoptosis was induced by either $\mathrm{H}_{2} \mathrm{O}_{2}$, heat shock or anti-Leishmania drugs such as miltefosine or curcumin. Even though LmjMCA has a functional N-terminal mitochondrial localisation signal, which is likely to be responsible for the partial LmjMCA mitochondrial localisation, the central catalytic domain alone was sufficient to enhance the parasite sensitivity to cell death indicating that the metacaspase would indirectly affect the mitochondrion to trigger cell death [37]. 


\begin{tabular}{|c|c|c|c|}
\hline & Structure, processing and enzymatic activity & Expression, localisation and function & References \\
\hline LdMC1/2 & $\begin{array}{l}\text { P-rich region in C-terminus } \\
\text { No processing with or without oxidative stress }\left(\mathrm{H}_{2} \mathrm{O}_{2}\right) \text {. } \\
\mathrm{R} / \mathrm{K} \text { specific proteolytic activity at } \mathrm{pH} 7.5 \text {, induced in } \mathrm{H}_{2} \mathrm{O}_{2} \\
\text { treated parasites. } \\
\text { Sensitive to leupeptin, antipain and TLCK but insensitive to } \\
\text { caspase inhibitors. } \\
\text { Absence of caspase-like protease activity. }\end{array}$ & $\begin{array}{l}\text { Expressed in both promastigote and axenic amastigote } \\
\text { parasites. } \\
\text { Colocalisation with the acidocalcisome compartments. } \\
\text { Parasites over-expressing LdMCs are more sensitive to } \\
\left(\mathrm{H}_{2} \mathrm{O}_{2}\right) \text {-induced cell death. }\end{array}$ & {$[36]$} \\
\hline LmjMCA & $\begin{array}{l}\text { P-rich region in C-terminus and signal peptide in } \mathrm{N} \text { - } \\
\text { terminus (functional MLS). } \\
\text { Autoprocessing and R-specific proteolytic activity } \\
\text { dependent on the } \mathrm{H} / \mathrm{C} \text { catalytic dyad. } \\
\text { Absence of caspase-like protease activity. } \\
\mathrm{LmjMCA} \text { was extensively processed and the central } \\
\text { catalytic domain accumulated in the cytosolic subcellular } \\
\text { fraction upon cell death induction. }\end{array}$ & $\begin{array}{l}\text { Expressed mainly in replicating amastigotes and procyclic } \\
\text { promastigotes but less in metacyclic promastigotes. } \\
\text { Localised in the nucleus during mitosis and in kinetoplast } \\
\text { during organelle segregation. } \\
\text { Promastigotes over-expressing LmjMCA suffered from } \\
\text { growth retardation and dysregulations in kinetoplast } \\
\text { segregation, nuclear division and cytokinesis. } \\
\Delta y c a 1 \text { yeast over-expressing LmjMCA are more sensitive } \\
\text { to }\left(\mathrm{H}_{2} \mathrm{O}_{2} \text { )-induced cell death. }\right. \\
\text { Parasites over-expressing LmjMCA showed mitochondrial } \\
\text { over-sensitivity to } \mathrm{H}_{2} \mathrm{O}_{2} \text {. }\end{array}$ & {$[35,37,38]$} \\
\hline
\end{tabular}

Interestingly, a non-death role has also been ascribed to LmjMCA when analyzed in its physiological context. LmjMCA was found to be expressed in actively replicating amastigotes and procyclic promastigotes, but to a lesser extent in metacyclics. It has a diffuse scattered distribution throughout the cell in interphase, becoming concentrated in the kinetoplast during organelle segregation and relocalising to the nucleus during mitosis. Here, it associates with the mitotic spindle, suggesting a role during organelle segregation and cell-cycle progression. Moreover, LmjMCA null mutants were only viable when LmjMCA was re-expressed from an episome at physiological levels, thus reinforcing its importance in parasite proliferation [38].

The discrepancies in metacaspase function and localisation range from a single metacaspase fulfilling several roles (as in L. major), to multiple metacaspases with structural and functional differences (as in T. brucei). From an evolutionary perspective, these could be of great interest as they could be used to trace the origin of the multiple roles fulfilled by the closely related caspases in metazoans.

\section{Targeting metacaspases to kill parasites}

Caspase inhibitors are under scrutiny by several groups working on infectious diseases in humans where apoptosis promotes tissue damage. Attempts were made to understand the mechanisms involved in caspase control in human cells, in order to control pathogen-induced apoptosis. Caspase inhibitors have been tested in preclinical studies with various models of acute or chronic liver failure. The pan-caspase inhibitor z-VAD-fmk decreased rat mortality after massive hepatectomy [39] and IDN-6556 reduced ischemia-reperfusion injury in rat $[40,41]$. This latter compound was used in proof-ofconcept trial with patient presenting viral hepatitis $C$
[42] and in human liver preservation injury trial [43]. These trials, leading to inhibit human cells apoptosis, are far from the goal to induce parasite apoptosis in vivo. However, this shows that apoptosis control could be one of the most stimulating challenges for the future. Inhibiting human cells apoptosis to avoid tissue injury could promote cancer if the intrinsic pathway is targeted. In contrast, stimulating parasite apoptosis to increase the anti-parasitic drug efficacy could promote human cell destruction from a similar mechanism.

Considering the specificity of metacaspases activity in protozoan parasites and their absence in humans, a new research area has opened to characterize metacaspase activators or inhibitors.

Recently, a series of inhibitors have been evaluated as competitive inhibitors of TbMCA2 and 3, and on T. brucei, T. cruzi, L. infantum and P. falciparum parasites cultures in vitro [44]. Activities against these protozoan parasites were in the micromolar range, far from what would be needed, but the high selectivity for TbMCA2 and TbMCA3, is a first interesting step.

Whether or not protozoan metacaspases are directly involved in parasite cell death per se, it could be speculated that their conservation in parasites presenting a long history of adaptation to environment implies that metacaspases are key regulators of the parasite life or death decision. Thus, metacaspases should be considered as potential drug target for the future. Consideration of trials in progress using drugs designed to facilitate apoptosis of cancer cells in humans leads to speculation that similar strategies could be used to induce protozoan death at different stages of their life cycles.

Thus, it is of utmost importance to explore deeply the pathways involved in parasite apoptosis, and the key regulatory proteins that mediate cell death. In human 
cells, these pathways are tightly regulated at multiple steps, including through phosphorylation [45]. For example, phosphorylation of human caspase 9 Thr125 by the cyclin-dependent kinase (CDK1) - cyclin B1 inhibits caspase 9 activation. That phosphorylation is inhibited by the protein kinase inhibitor staurosporine. Additional sites of phosphorylation in caspase 9 were also reported (Ser 196, Ser 144, Ser 183, Ser195, Tyr 153, etc.) with different effects on cell apoptosis [45]. It could thus be speculated that parasite metacaspases could also be regulated by phosphorylation at specific stages of parasite cycles, and this should be taken into account to provide definitive data on the role of metacaspase in parasite death and as a drug target.

Finally, the high homology among protozoan metacaspases and the fact that these proteins are not present in mammals make them good candidates as drug targets to fight parasitic diseases. A future drug should be designed to activate the metacaspase pathway in order to favour parasite death, either directly or through the inhibition of one of the numerous negative controls suspected to be present in fast growing parasites. Inhibition of human cells apoptosis induced by parasitic diseases [46] combined with induction of parasite apoptosis at the initial steps of the infection is an integrative concept for future treatment that still needs to be proven. In both cases, recent breakthroughs in knowledge allow reasonable optimism.

\section{Acknowledgements}

The authors are members of COST action BM0802 'Life and death of protozoan parasites' and appreciate support from this action.

\section{Author details}

${ }^{1}$ Malaria Research Unit, University Lyon 1, 8 Avenue Rockefeller, 69373 Lyon cedex 08, France. ${ }^{2}$ Department of Biochemistry, University of Lausanne, 155 Chemin des Boveresses, 1066 Epalinges, Switzerland.

\section{Authors' contributions}

All authors contributed equally to the draft of the manuscript. All authors read and approved the final manuscript.

\section{Competing interests}

The authors declare that they have no competing interests.

Received: 28 October 2010 Accepted: 28 February 2011

Published: 28 February 2011

\section{References}

1. Aravind L, Dixit VM, Koonin EV: The domains of death: evolution of the apoptosis machinery. Trends Biochem Sci 1999, 24:47-53.

2. Uren AG, O'Rourke K, Aravind LA, Pisabarro MT, Seshagiri S, Koonin EV Dixit VM: Identification of paracaspases and metacaspases: two ancient families of caspase-like proteins, one of which plays a key role in MALT lymphoma. Mol Cell 2000, 6:961-967.

3. Madeo F, Herker E, Maldener C, Wissing S, Lächelt S, Herlan M, Fehr M, Lauber K, Sigrist SJ, Wesselborg S, Fröhlich KU: A caspase-related protease regulates apoptosis in yeast. Mol Cell 2002, 9:911-7.

4. Khan MA, Chock PB, Stadtman ER: Knockout of caspase-like gene, YCA1, abrogates apoptosis and elevates oxidized proteins in Saccharomyces cerevisiae. Proc Natl Acad Sci USA 2005, 102(173):26-31.
5. Mazzoni C, Falcone C: Caspase-dependent apoptosis in yeast. Biochim Biophys Acta 2008, 783:1320-7.

6. Váchová L, Palková Z: Caspases in yeast apoptosis-like death: facts and artefacts. FEMS Yeast Res 2007, 7:12-21.

7. Lee RE, Puente LG, Kaern M, Megeney LA: A non-death role of the yeast metacaspase: Yca1p alters cell cycle dynamics. PLoS One 2008, 3:e2956.

8. Enoksson M, Salvesen GS: Metacaspases are not caspases-always doubt. Cell Death Differ 2010, 17:1221.

9. Vercammen D, Declercq W, Vandenabeele P, Van Breusegem F: Are metacaspases caspases? J Cell Biol 2007, 179:375-380.

10. Carmona-Gutierrez D, Fröhlich KU, Kroemer G, Madeo F: Metacaspases are caspases. Doubt no more. Cell Death Differ 2010, 17:377-8.

11. Rawlings ND: Peptidase inhibitors in the MEROPS database. Biochimie 2010, 92:1463-83.

12. Atkinson HJ, Babbitt PC, Sajid M: The global cysteine peptidase landscape in parasites. Trends Parasitol 2009, 25:573-581.

13. Mottram JC, Helms MJ, Coombs GH, Sajid M: Clan CD cysteine peptidases of parasitic protozoa. Trends Parasitol 2003, 19:182-187.

14. Coll NS, Vercammen D, Smidler A, Clover C, Van Breusegem F, Dangl JL, Epple P: Arabidopsis type I metacaspases control cell death. Science 2010, 330:1393-7.

15. Belenghi B, Romero-Puertas MC, Vercammen D, Brackenier A, Inzé D, Delledonne M, Van Breusegem F: Metacaspase activity of Arabidopsis thaliana is regulated by S-nitrosylation of a critical cysteine residue. J Biol Chem 2007, 282:1352-1358.

16. Sundström JF, Vaculova A, Smertenko AP, Savenkov El, Golovko A, Minina E, Tiwari BS, Rodriguez-Nieto S, Zamyatnin AA Jr, Välineva T, Saarikettu J, Frilander MJ, Suarez MF, Zavialov A, Ståhl U, Hussey PJ, Silvennoinen O, Sundberg E, Zhivotovsky B, Bozhkov PV: Tudor staphylococcal nuclease is an evolutionarily conserved component of the programmed cell death degradome. Nat Cell Biol 2009, 11:1347-54.

17. Hastings I: How artemisinin-containing combination therapies slow the spread of antimalarial drug resistance. Trends Parasitol 2011, 27:67-72.

18. Picot S, Burnod J, Bracchi V, Chumpitazi BF, Ambroise-Thomas P: Apoptosis related to chloroquine sensitivity of the human malaria parasite Plasmodium falciparum. Trans R Soc Trop Med Hyg 1997, 91:590-591.

19. Al-Olayan EM, Williams GT, Hurd H: Apoptosis in the malaria protozoan, Plasmodium berghei: a possible mechanism for limiting intensity of infection in the mosquito. Int J Parasitol 2002, 32:1133-1143.

20. Wu Y, Wang $X$, Liu $X$, Wang Y: Data-mining approaches reveal hidden families of proteases in the genome of malaria parasite. Genome Res 2003, 13:601-616.

21. Rosenthal PJ: Cysteine proteases of malaria parasites. Int J Parasito/ 2004 34:1489-1499.

22. Meslin B, Barnadas C, Boni V, Latour C, De Monbrison F, Kaiser K, Picot S Features of apoptosis in Plasmodium falciparum erythrocytic stage through a putative role of PfMCA1 metacaspase-like protein. J Infect Dis 2007, 195:1852-1859.

23. Le Chat L, Sinden RE, Dessens JT: The role of metacaspase 1 in Plasmodium berghei development and apoptosis. Mol Biochem Parasitol 2007, 153:41-47.

24. Ali M, Al-Olayan EM, Lewis S, Matthews $H$, Hurd H: Naturally occurring triggers that induce apoptosis-like programmed cell death in Plasmodium berghei ookinetes. PLoS One 2010, 5:e12634.

25. Mutai BK, Waitumbi JN: Apoptosis stalks Plasmodium falciparum maintained in continuous culture condition. Malaria J 2010, 9(Suppl 3):S6.

26. Hossain MJ, Korde R, Singh S, Mohmmed A, Dasaradhi PV, Chauhan VS, Malhotra P: Tudor domain proteins in protozoan parasites and characterization of Plasmodium falciparum tudor staphylococcal nuclease. Int J Parasitol 2008, 38:513-26.

27. Hossain MJ, Korde R, Singh PK, Kanodia S, Ranjan R, Ram G, Kalsey GS, Singh R, Malhotra P: Plasmodium falciparum Tudor Staphylococcal Nuclease interacting proteins suggest its role in nuclear as well as splicing processes. Gene 2010, 468:48-57.

28. Lescure FX, Le Loup G, Freilij H, Develoux M, Paris L, Brutus L, Pialoux G: Chagas disease: changes in knowledge and management. Lancet Infect Dis 2010, 10:556-70.

29. Den Boer ML, Alvar J, Davidson RN, Ritmeijer K, Balasegaram M: Developments in the treatment of visceral leishmaniasis. Expert Opin Emerg Drugs 2009, 14:395-410. 
30. Helms MJ, Ambit A, Appleton P, Tetley L, Coombs GH, Mottram JC: Bloodstream form Trypanosoma brucei depend upon multiple metacaspases associated with RAB11-positive endosomes. J Cell Sci 2006, 119:1105-1117.

31. Szallies A, Kubata BK, Duszenko M: A metacaspase of Trypanosoma brucei causes loss of respiration competence and clonal death in the yeast Saccharomyces cerevisiae. FEBS Lett 2002, 517:144-150.

32. Moss CX, Westrop GD, Juliano L, Coombs GH, Mottram JC: Metacaspase 2 of Trypanosoma brucei is a calcium-dependent cysteine peptidase active without processing. FEBS Lett 2007, 581:5635-5639.

33. Watanabe N, Lam E: Two Arabidopsis metacaspases AtMCP1b and AtMCP2b are arginine/lysine-specific cysteine proteases and activate apoptosis-like cell death in yeast. J Biol Chem 2005, 280:14691-14699.

34. Kosec G, Alvarez VE, Agüero F, Sánchez D, Dolinar M, Turk B, Turk V, Cazzulo JJ: Metacaspases of Trypanosoma cruzi: possible candidates for programmed cell death mediators. Mol Biochem Parasitol 2006, 145:18-28.

35. González IJ, Desponds C, Schaff C, Mottram JC, Fasel N: Leishmania major metacaspase can replace yeast metacaspase in programmed cell death and has arginine-specific cysteine peptidase activity. Int J Parasitol 2007 37:161-172.

36. Lee N, Gannavaram S, Selvapandiyan A, Debrabant A: Characterization of metacaspases with trypsin-like activity and their putative role in programmed cell death in the protozoan parasite Leishmania. Eukaryot Cell 2007, 6:1745-1757.

37. Zalila H, González IJ, El-Fadili A, Delgado M, Desponds C, Schaff C, Fasel N: Processing of metacaspase into a cytoplasmic catalytic domain mediating cell death in Leishmania major. Mol Microbiology 2011, 79:222-39.

38. Ambit A, Fasel N, Coombs GH, Mottram JC: An essential role for the Leishmania major metacaspase in cell cycle progression. Cell Death Differ 2008, 15:113-122.

39. Yoshida N, Iwata H, Yamada T, Sekino T, Matsuo H, Shirahashi K, Miyahara T, Kiyama S, Takemura H: Improvement of the survival rate after rat massive hepatectomy due to the reduction of apoptosis by caspase inhibitor. $J$ Gastroenterol Hepatol 2007, 22:2015-2021.

40. Natori S, Selzner M, Valentino KL, Fritz LC, Srinivasan A, Clavien PA, Gores GJ: Apoptosis of sinusoidal endothelial cells occurs during liver preservation injury by a caspase-dependent mechanism. Transplantation 1999, 68:89-96.

41. Hoglen NC, Anselmo DM, Katori M, Kaldas M, Shen XD, Valentino KL, Lassman C, Busuttil RW, Kupiec-Weglinski JW, Farmer DG: A caspase inhibitor, IDN-6556, ameliorates early hepatic injury in an ex vivo rat model of warm and cold ischemia. Liver Transpl 2007, 13:361-366.

42. Pockros PJ, Schiff ER, Shiffman ML, McHutchison JG, Gish RG, Afdhal NH, Makhviladze M, Huyghe M, Hecht D, Oltersdorf T, Shapiro DA: Oral IDN6556, an antiapoptotic caspase inhibitor, may lower aminotransferase activity in patients with chronic hepatitis C. Hepatology 2007, 46:324-329.

43. Baskin-Bey ES, Washburn K, Feng S, Oltersdorf T, Shapiro D, Huyghe M, Burgart L, Garrity-Park M, van Vilsteren FG, Oliver LK, Rosen CB, Gores GJ: Clinical Trial of the Pan-Caspase Inhibitor, IDN-6556, in Human Liver Preservation Injury. Am J Transplant 2007, 7:218-225.

44. Berg M, Van der Veken $P$, Joossens J, Muthusamy V, Breugelmans $M$, Moss CX, Rudolf J, Cos P, Coombs GH, Maes L, Haemers A, Mottram JC, Augustyns K: Design and evaluation of Trypanosoma brucei metacaspase inhibitors. Bioorg Med Chem Lett 2010, 20:2001-2006.

45. Allan LA, Clarke PR: Apoptosis and autophagy: Regulation of caspase- 9 by phosphorylation. FEBS J 2009, 276:6063-6073.

46. Bienvenu AL, Gonzalez-Rey E, Picot S: Apoptosis induced by parasitic diseases. Parasite Vectors 2010, 17(3):106.

\section{doi:10.1186/1756-3305-4-26}

Cite this article as: Meslin et al:: Are protozoan metacaspases potential parasite killers? Parasites \& Vectors 2011 4:26.

\section{Submit your next manuscript to BioMed Central and take full advantage of:}

- Convenient online submission

- Thorough peer review

- No space constraints or color figure charges

- Immediate publication on acceptance

- Inclusion in PubMed, CAS, Scopus and Google Scholar

- Research which is freely available for redistribution

Submit your manuscript at www.biomedcentral.com/submit
Biomed Central 